\title{
ARTICLE OPEN Tracking the evolution of intergranular corrosion through twin-related domains in grain boundary networks
}

\author{
Christopher M. Barr ${ }^{1}$, Sebastian Thomas ${ }^{2}$, James L. Hart ${ }^{1}$, Wayne Harlow ${ }^{1}$, Elaf Anber ${ }^{1}$ and Mitra L. Taheri ${ }^{1}$
}

Tailoring the grain boundary network is desired to improve grain boundary-dependent phenomena such as intergranular corrosion. An important grain boundary network descriptor in heavily twinned microstructures is the twin-related domain, a cluster of twinrelated grains. We indicate the advantages of using twin-related domains and subsequent statistics to provide new insight into how a grain boundary networks respond to intergranular corrosion in a heavily twinned grain boundary engineered $316 \mathrm{~L}$ stainless steel. The results highlight that intergranular corrosion is typically arrested inside twin-related domains at coherent twins or low-angle grain boundaries. Isolated scenarios exist, however, where intergranular corrosion propagation persists in the grain boundary network through higher-order twin-related boundaries.

npj Materials Degradation (2018)2:14; doi:10.1038/s41529-018-0032-7

\section{INTRODUCTION}

Austenitic stainless steels including 304/304L and 316 L are critical structural alloys used in a diverse number of engineering applications due to a combination of good mechanical properties, weldability, and overall corrosion resistance. However, intergranular corrosion (IGC) in austenitic stainless steels and other alloys can have a devastating effect on many of these industries, including nuclear, naval ship structures, high temperature processing equipment, and off-shore wind turbines. IGC in austenitic stainless steels is linked to the sensitization of the grain boundary (GB) network. GB sensitization can occur during welding and heat treatment in the $500-800{ }^{\circ} \mathrm{C}$ temperature regime and results in the precipitation and growth of deleterious GB Cr-rich carbides and subsequent $\mathrm{Cr}$-depleted zones. $^{1-3}$ Significant effort has been made to mitigate the deleterious effects of sensitization by introducing a high density of GBs resistant both $\mathrm{Cr}$-rich carbide formation and IGC through grain boundary engineering (GBE). ${ }^{4-10}$ GBE is the process of introducing a specific type and density of GBs through thermomechanical processing (TMP) to improve a GB-dependent property. Previous studies have shown GBE to be a successful method in mitigating intergranular stress corrosion cracking, $^{11,12} \mathrm{IGC}^{5,13,14}$ hydrogen embrittlement, ${ }^{15}$ and grain growth. ${ }^{16}$

GBE is typically associated with the increase in specific coincidence site lattice (CSL) GBs, which have been considered special boundaries. ${ }^{4}$ Early research within the field of GBE described some or all low $\Sigma$ CSL GBs as special. It is apparent, however, that low $\Sigma$ CSL boundaries cannot exclusively describe low energy, low excess volume, or special GB properties. ${ }^{17}$ The full GB character distribution, including GB plane normal and misorientation, is required to describe the macroscopic GB structure. Nevertheless, the use of the term "special" or low $\Sigma$ CSL boundaries is convenient, and thus GBE is most often related to the large increases seen in the fraction of twin $(\Sigma 3)$, both coherent and non-coherent, and twin-related GBs, $\Sigma 3^{n}(n>1)$ in low to medium stacking fault materials such as Ni-based alloys, austenitic stainless steels, and copper after TMP.

A more recent approach to the characterization of GB networks in heavily twinned microstructures is the use of the following descriptors: twin-related grain clusters or twin-related domains (TRD). ${ }^{18-23}$ Gertsman et al. ${ }^{24}$ defined a twin-related grain cluster as a region of a microstructure in which all of the GBs within a given cluster of grains are described by a $\Sigma 3^{n}(n \geq 1)$ misorientation. Fang et al. ${ }^{25}$ and Xia et al. ${ }^{26}$ implement the grain cluster analysis in studies of GBE stainless steel and Ni-based Alloy 690, respectively. Hu et al. ${ }^{5}$ examined grain clusters of related $\Sigma 3^{n} \mathrm{GBs}$ in engineered 316 stainless steel and found that only $\Sigma 3$ coherent twins $(\Sigma 3 c)$ inside the grain cluster are resistant to IGC. In a similar fashion to the twin grain cluster studies above, Reed et al. ${ }^{27}$ and Cayron ${ }^{28}$ described a TRD as a large cluster of twin-related grains. Reed et al. ${ }^{29}$ show that TRDs between an as-received Ni-based Alloy 600 have simple twinning and are isolated from the random highangle GB network while, in contrast, the TRDs of GBE condition possess a high density of twin-related GBs and a break-up of the random GB network. While these studies showed that TRDs are relevant GB network descriptor for heavily twinned microstructures, there has been limited correlation between TRD statistics and IGC response of different TMP microstructures.

In this study, we provide insight into how TRD statistic between a GBE and solution anneal (SA) condition can be used to effectively understand and correlate IGC phenomena. Furthermore, the manuscript highlights how IGC propagation and arrest occur in the context of TRDs in the heavily twinned GBE condition. We observe that twin-related GBs inside TRDs effectively stop or arrest IGC propagation due to triple junctions in the domain interior that contain either (1) two $\Sigma 3$ coherent boundaries, or (2) a $\Sigma 3$ coherent boundary and a low-angle GB. The analysis highlights the observation that despite the TRDs' ability to stop corrosion propagation, particular situations exist, due to higher-order noncorrosion-resistant twin-related GBs, which allow corrosion

\footnotetext{
${ }^{1}$ Materials Science and Engineering Department, Drexel University, 3141 Chestnut Street, Philadelphia, PA 19104, USA and ${ }^{2}$ Department of Materials Science and Engineering, Monash University, 22 Alliance Lane, Clayton, VIC 3800, Australia

Correspondence: Mitra L. Taheri (mtaheri@coe.drexel.edu)
}

Received: 6 August 2017 Revised: 30 January 2018 Accepted: 5 February 2018

Published online: 03 April 2018 
damage to run through the length or middle of the TRD. Therefore, not only is it important to distinguish between coherent and non-coherent $\Sigma 3$, but also, it is critical to delineate higher-order twin boundaries from one another, all of which occur inside the TRD. To complement the TRD network IGC analysis between a GBE and non-GBE microstructure, double loopelectrochemical potentiokinetic reactivation (DL-EPR) and intergranular mass loss testing was also completed to determine the degree of sensitization (DOS) after sensitization heat treatments. The DL-EPR method has been widely used to quantitatively examine DOS and IGC susceptibility in stainless steels. ${ }^{30-33}$ Overall, the study shows that using TRDs as an effective and useful microstructural GB network descriptor provide enhanced insight into individual $G B$ and $G B$ network IGC phenomena in GBE stainless steel.

\begin{tabular}{|lll|}
$\begin{array}{l}\text { Table } 1 . \quad \text { Distribution of triple junction types for the SA and GBE } \\
\text { condition }\end{array}$ \\
\hline Triple junction type distribution & $\mathrm{SA}(\%)$ & $\mathrm{GBE}(\%)$ \\
\hline$\Sigma 3 \mathrm{C}, \Sigma 3 \mathrm{c}, \mathrm{R}$ & 0.9 & 0.8 \\
$\Sigma 3 \mathrm{C}, \Sigma 3 \mathrm{c}, \Sigma 9$ & 2.6 & 9.5 \\
$\Sigma 3 \mathrm{C}, \Sigma 9, \Sigma 27$ & 0.4 & 7.6 \\
$\Sigma 3 \mathrm{C}, \mathrm{R}, \mathrm{R}$ & 50.9 & 32.5 \\
$\Sigma 3 \mathrm{i}, \Sigma 3 \mathrm{i}, \mathrm{R}$ & 0.5 & 0.8 \\
$\Sigma 3 \mathrm{i}, \Sigma 3 \mathrm{i}, \Sigma 9$ & 0.2 & 2.2 \\
$\Sigma 3 \mathrm{i}, \Sigma 9, \Sigma 27$ & 0.1 & 2.7 \\
$\Sigma 3 \mathrm{i}, \mathrm{R}, \mathrm{R}$ & 8.7 & 9.8 \\
$\Sigma 3 \mathrm{i}, \Sigma 3 \mathrm{c}, \mathrm{R}$ & 3 & 2.5 \\
$\Sigma 3 \mathrm{i}, \Sigma 3 \mathrm{C}, \Sigma 9$ & 1.1 & 10.1 \\
\hline
\end{tabular}

\section{RESULTS}

$\mathrm{GB}$ and triple junction type distribution

The $\Sigma 3$ GB length fraction including coherent and non-coherent twin segments increased from 47 to $68 \%$, while the $\Sigma 9+\Sigma 27$ combined GB length fraction increased from 0.4 to $8.8 \%$ from the $\mathrm{SA}$ to GBE condition. The increases in the $\Sigma 3$ and $\Sigma 3^{n}(n>1)$ length fraction observed after TMP are consistent with previously reported GBE processing in 304 or 316 type stainless steels. $5,34,35$ Table 1 provides the distribution of triple junction types, a descriptor of GB network connectivity, for both conditions. There is a significant increase in triple junctions containing two $\Sigma 3 \mathrm{GBs}$ from the SA to GBE condition. The distribution of GBs in a triple junction, while offering an overview of how different GBs are connected in the overall GB network, does not provide a method to understand local GB connectivity related to IGC.

\section{Twin-related domains}

TRDs for the GBE and SA condition are shown in Fig. 1a and c, where a number identifies a unique grain orientation. Figure $1 b$ and $d$ shows the corresponding twinning tree for the GBE and SA TRDs where each unique grain orientation is classified as a node and are linked exclusively by $\Sigma 3$ relationship. In total, the TRD examined in this study for the GBE condition had 45 distinct orientations, whereas the SA condition had three distinct orientations. All GBs inside the TRD interior are twin-related GBs $\left(\Sigma 3^{n}, n \geq 1\right)$ and have less than $2^{\circ}$ deviation from their ideal CSL misorientation. A large fraction of these GBs are $\Sigma 3, \Sigma 9$, or $\Sigma 27$ and are represented by red $(\Sigma 3)$ and blue $(\Sigma 9, \Sigma 27)$ colored lines in Fig. 1. Other high and low-angle GBs exist in TRDs and are represented by black (high-angle GB) and purple (low-angle GB) colored lines which correspond to $\Sigma 3^{4}$ or higher-order twin relationships. Important for IGC analysis and GB connectivity, low-angle GBs can form through multiple twinning events. Two low-angle GBs that

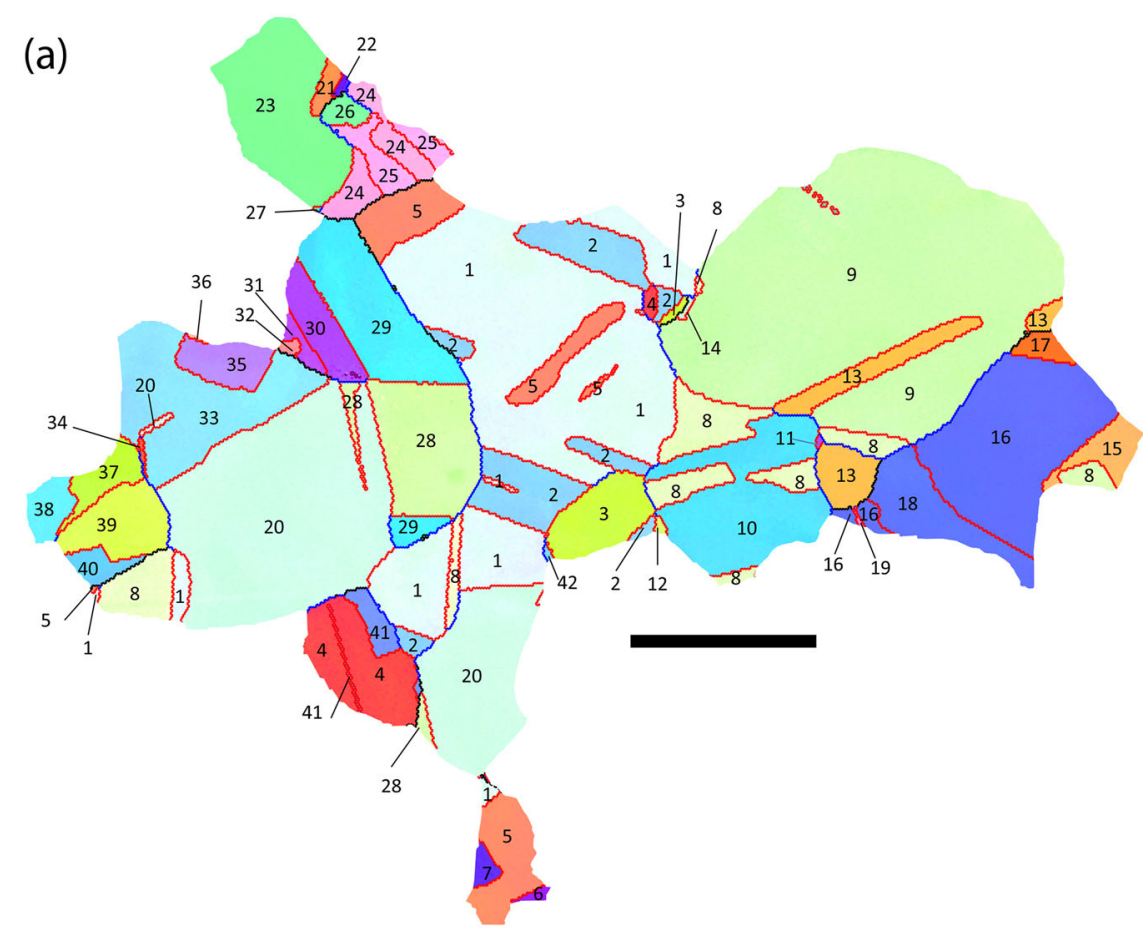

(b)

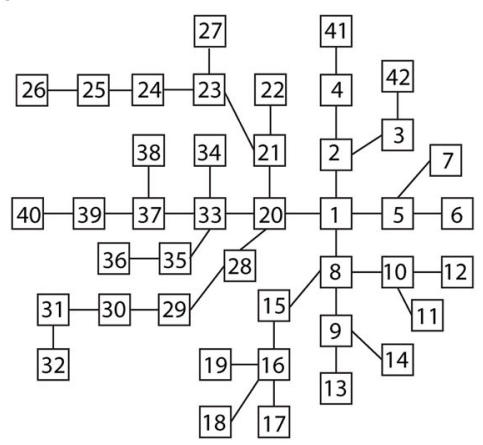

(c)

(d)

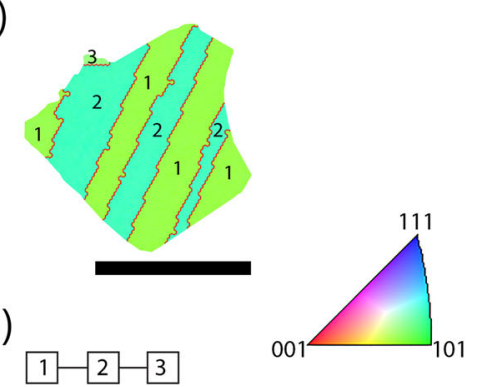

Fig. 1 a Representative TRD for the GBE condition where each orientation is given a number, while the grain color is based on the inverse pole figure scheme. $\mathbf{b}$ Corresponding TRD twinning tree from a where each orientation is linked by a $\Sigma 3$ relationship. $\mathbf{c}$ Representative TRD for the SA condition where each orientation is given a number, while the grain color is based on the inverse pole figure scheme. $\mathbf{d}$ Corresponding TRD twinning tree from $\mathrm{c}$ where each orientation is linked by a $\Sigma 3$ relationship. In the TRD maps a and c, $\Sigma 3 \mathrm{GBs}$ are colored red, $\Sigma 9$ and $\Sigma 27$ GBs are colored blue, while high-angle and low-angle GBs are colored black and purple, respectively. Scale bar in a and c are 200 and $75 \mu \mathrm{m}$, respectively 

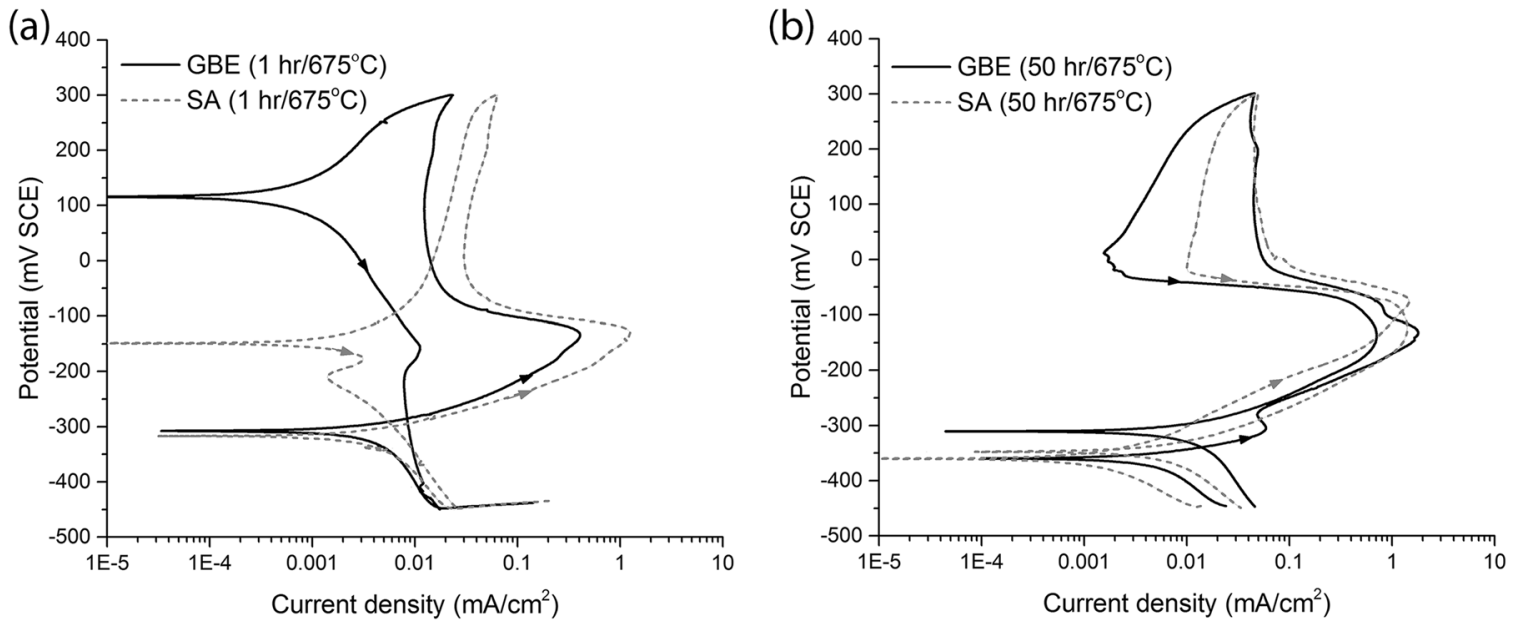

Fig. 2 DL-EPR curves for the SA and GBE conditions for both a $1 \mathrm{~h}$ sensitization heat treatment and $\mathbf{b} 50 \mathrm{~h}$ sensitization heat treatment. The DOS can be estimated from the peak current densities of the forward and reverse scans, using Eq. 1

occur in GBE TRDs are: $12.2^{\circ}<311>$ and $7.36^{\circ}<110>$ which are both $\Sigma 243\left(\Sigma 3^{5}\right)$ relationships. ${ }^{36}$ The ability to analyze the GB network with the TRD twinning tree provides a more expansive and detailed overview of the GB character. Specifically, the twinning tree provides the ability to track higher-order $\Sigma 3$ relationships in the TRD.

\section{Connecting IGC to TRD statistics}

Figure $2 \mathrm{a}$ shows the representative DL-EPR curves for the SA and GBE conditions after the $1 \mathrm{~h}$ and $50 \mathrm{~h} 675^{\circ} \mathrm{C}$ sensitization heat treatment. A higher DOS (2.7\%) is observed for the SA condition compared to the DOS $(0.25 \%)$ obtained in the GBE condition for the $1 \mathrm{~h}$ sensitization condition. The $1 \mathrm{~h}$ sensitization condition of the GBE condition indicates a lack of consistent reactivation peak $\left(I_{r}\right)$, which correlates with samples having a minimal density of $\mathrm{Cr}$ rich carbides and subsequently chromium-depleted zones along the GB network. The SA condition with a $2.7 \%$ DOS indicates a low to moderate DOS value which is also consistent with the relatively short sensitization heat treatment for low carbon $\mathrm{Fe}, \mathrm{Ni}, \mathrm{Cr}$, and Mo containing stainless steels such as $316 \mathrm{~L}$. The $50 \mathrm{~h}$ sensitization heat treatment, shown in Fig. 2b, indicates higher DOS for both the SA and GBE conditions. The DOS for the SA condition was found to be nearly double that of the GBE condition. Specifically, the average DOS for the SA and GBE conditions was 95 and $46.5 \%$, respectively. The high DOS in the SA condition is consistent with significant $G B$ attack due to the formation of $\mathrm{Cr}$-rich carbides and subsequent GB Cr-depleted zones during sensitization.

Figure 3 shows the IGC response and corrosion rate for the SA and GBE conditions for both the 1 and $50 \mathrm{~h}$ sensitization conditions during $120 \mathrm{~h}$ exposure to the FS-SA ASTM 262 Practice $B$ test. The GBE condition has significantly reduced corrosion rates in both the low sensitization and high sensitization regime compared to the SA condition. As expected, the corrosion rate and IGC attack is more significant after $50 \mathrm{~h}$ at $675^{\circ} \mathrm{C}$ in both the GBE and SA condition. The low and high sensitization treatment in the SA sample has a high degree of connected IGC regions, while the GBE sample has more scattered, disjointed IGC regions. Unlike the GBE condition in the $50 \mathrm{~h}$ sensitization treatment, the SA condition visually indicates the presence of significant grain drop out, a key indication of a lack IGC resistance.

In order to validate using TRDs as an effective microstructural descriptor to evaluate IGC in heavily twinned microstructures, Table 2, reproduced from a previous publication from the authors, ${ }^{23}$ outlines TRD statistics from the SA and GBE conditions. Specifically, the number of unique orientations, TRD average size, and the ratio of TRD to grain size provide a statistical examination of the variation in TRDs between the GBE and SA condition. The GBE condition indicates a significant increase in the TRD to grain size ratio. This TRD to grain size ratio indicates that the GBE condition, in comparison to the SA, contains spatially large TRDs but also contains twin limited small grains within the TRD. Table 2 further indicates that the overall TRD size, measured as the effective TRD circular diameter from 50 individual TRDs, shows a substantial increase from the SA to GBE condition. Overall, the IGC bulk testing and correlated TRD statistics indicate that microstructures that contain larger TRDs with a high density of $\Sigma 3$ GBs in the TRD interior have improved IGC resistance. Figure $3 e$ and $f$ shows a proposed schematic of how the size of the overall TRD can be a descriptor of the observed IGC behavior. The small TRDs observed in the SA condition have a high degree of connected and corroded GBs, while the large TRDs observed in the GBE condition have a discontinuous network of corroded GBs.

Propagation of IGC through TRDs. Figure 4 indicates the propagation of IGC at the exterior of two TRDs in the GBE condition. Figure $4 a$ and $b$ shows the SEM and inverse pole figure colored map images, respectively, of the same spatial location between two TRDs. The exterior of the TRDs in Fig. $4 a$ and $b$ are indicated by the orange line in the schematic of Fig. 4c. The image highlights that these non-twin-related random GBs at the exterior of the TRD are typically susceptible to IGC. In order to understand how IGC propagates into the TRD, it is important to explore the triple junctions along the exterior of the TRD. Figure 4 shows 14 triple junctions along the TRD exterior. All triple junctions at TRD exterior contain two random GBs and one twin-related GB. The IGC connects through the random GBs belonging to both adjacent triple junctions. In 9 of 14 triple junctions, the 3rd GB is a coherent twin. At these triple junctions, IGC propagation into the TRD is promptly arrested at the coherent twin GBs. The remaining 5 of the 14 triple junctions contain one of either a high-order twin GB $\left(\Sigma 3^{n} n>1\right)$ or a $\Sigma 3$ non-coherent twin. At these triple junctions, IGC is observed to propagate into the interior of the TRD.

While IGC is observed to propagate into TRDs through susceptible higher-order $\sum 3^{n}(n>1)$ or non-coherent $\Sigma 3$ twins, Fig. 5 highlights how propagation is typically arrested inside the TRD at a triple junction containing two coherent twins. Figure 5 further illustrates how the propagation continues through most of the higher-order twin-related GBs. In Fig. 5, IGC propagates first through the GB labeled A1 which is a $\Sigma 81 \mathrm{~d}, 60.1^{\circ}<443>\mathrm{GB}$. IGC attack continues into the TRD through several other GBs labeled A2 through A7, which includes $\Sigma 81 \mathrm{~d}, \Sigma 27 \mathrm{a}$, and $\Sigma 9 \mathrm{GBs}$. In this 

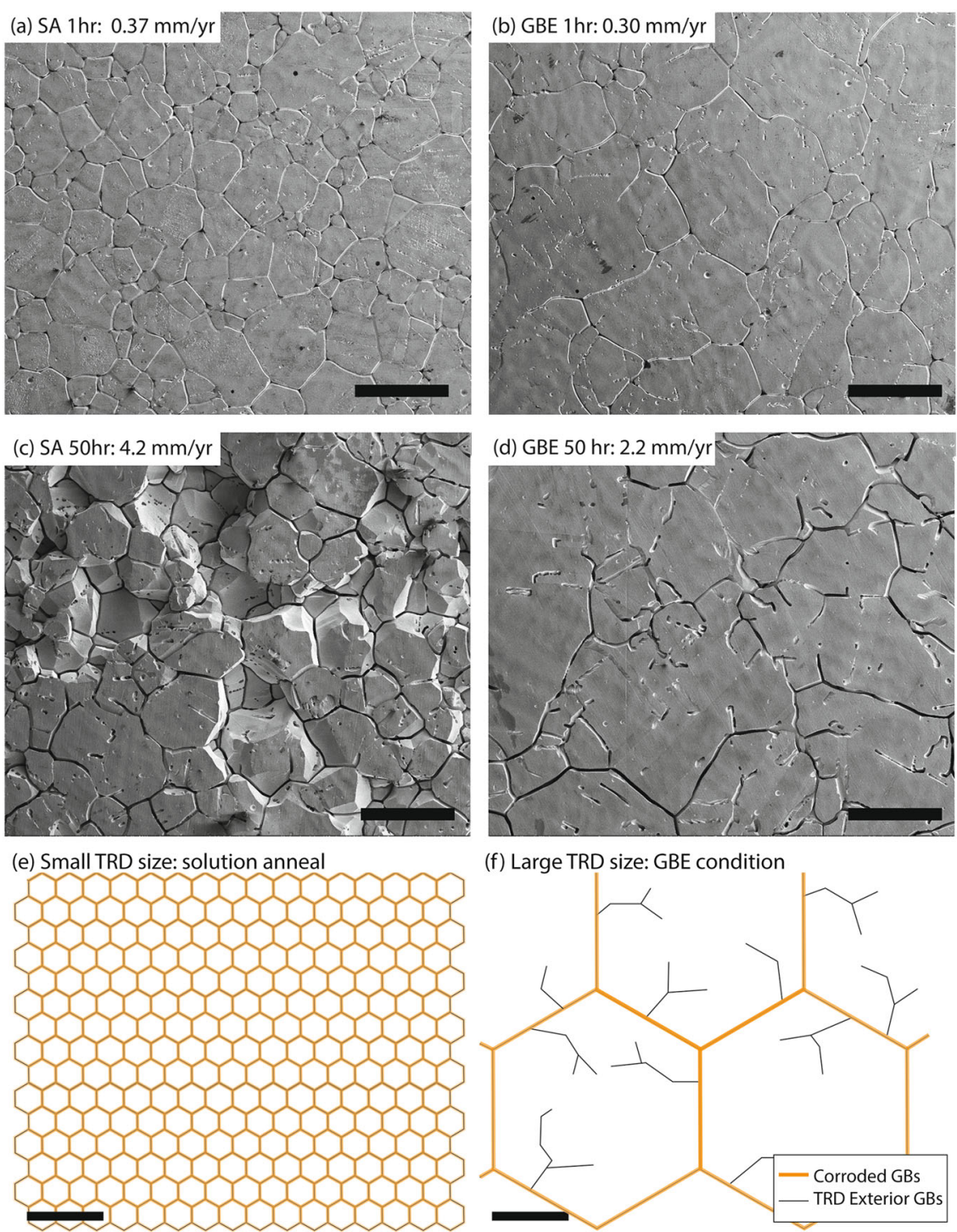

Fig. 3 IGC observed after $120 \mathrm{~h}$ in the ASTM A262 test for the a SA condition with $1 \mathrm{~h}$ sensitization, b GBE condition with $1 \mathrm{~h}$ sensitization, c SA condition with $50 \mathrm{~h}$ sensitization, d GBE condition with $50 \mathrm{~h}$ sensitization. e-f Schematic illustrating observed correlation between TRD size and corroded GBs in the SA and GBE condition. Scale bar is $200 \mu \mathrm{m}$

Table 2. TRD microstructural statistics relevant to IGC for the SA and GBE condition

\begin{tabular}{llll}
\hline TMP condition & Unique orientations & $\begin{array}{l}\text { TRD size } \\
(\mu \mathrm{m})\end{array}$ & $\begin{array}{l}\text { TRD to grain size } \\
\text { ratio }\end{array}$ \\
\hline SA & $2.4 \pm 1.4$ & 69 & 1.5 \\
GBE & $14.1 \pm 11.5$ & 396 & 4.7 \\
\hline Note: Data reproduced from previous publication ${ }^{23}$ & \\
\hline
\end{tabular}

example, IGC is continuous through a series of six triple junctions that contain two $\Sigma 3^{n}(n>1)$, which are not corrosion resistant, and one corrosion-resistant coherent twin boundary. As described above, the continuous IGC attack is stopped when it reaches a triple junction containing two coherent $\Sigma 3$ GBs labeled A8. Figure 5 also shows clear IGC on a non-coherent twin segment which is labeled A9. From this example, which is representative of TRDs in the GBE condition, triple junctions that contain two coherent twins are shown to be highly effective in stopping IGC propagation.
Since every unique orientation and subsequently every $\Sigma 3^{n} \mathrm{~GB}$ relationship is identified in the TRD and twinning tree (i.e., Fig. 1), it is possible to explore if all higher-order twin relationships are susceptible to IGC. It is found that during the GBE process and multiple twinning, two low-angle twin-related GBs, $\Sigma 243 \mathrm{C}\left(12.2^{\circ}\right.$ $<311>$ ) and $\Sigma 243 \mathrm{i}\left(7.36^{\circ}<110>\right)$, can form in the GB network. Figure 6 provides one example for the $\Sigma 243 i$ GB in which IGC propagation is arrested. While this is a different TRD than shown in previous examples, it clearly illustrates that low-angle GBs are both created by multiple twinning and are typically resistant to IGC. In Fig. 6, IGC propagation is seen at GBs labeled B1 and B2 inside the TRD which are $\Sigma 27 \mathrm{a}$ and $\Sigma 81 \mathrm{~d}$ GBs, respectively. The IGC propagation stops when reaching the triple junction that contains GBs labeled B6 and B3, which are a coherent twin and a $\Sigma 243 \mathrm{i} \mathrm{low-}$ angle $\mathrm{GB}$, respectively.

While IGC propagation is typically arrested within the TRD, scenarios exist in which IGC can fully propagate throughout the TRD. Figure 7 provides an example where IGC continuously exists throughout a significant section of the TRD shown in Fig. 1. In this example, all the GBs are higher-order high-angle twin-related GBs. Subsequently, all interconnected triple junctions in this case have 



(c)

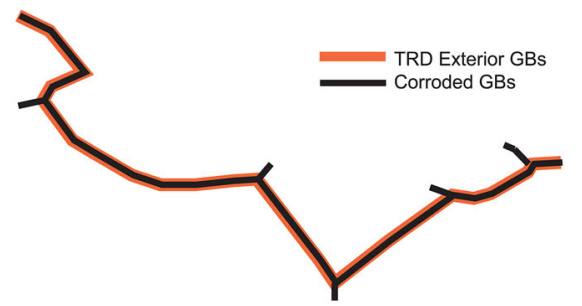

Fig. 4 a SEM image after IGC where corroded GBs show visible attack along all GBs at the TRD exterior. b Corresponding inverse pole figure colored map with GB type overlaid at same location in a. c Diagram illustrating that IGC is continuous along all the TRD exterior GBs. Scale bar is $50 \mu \mathrm{m}$
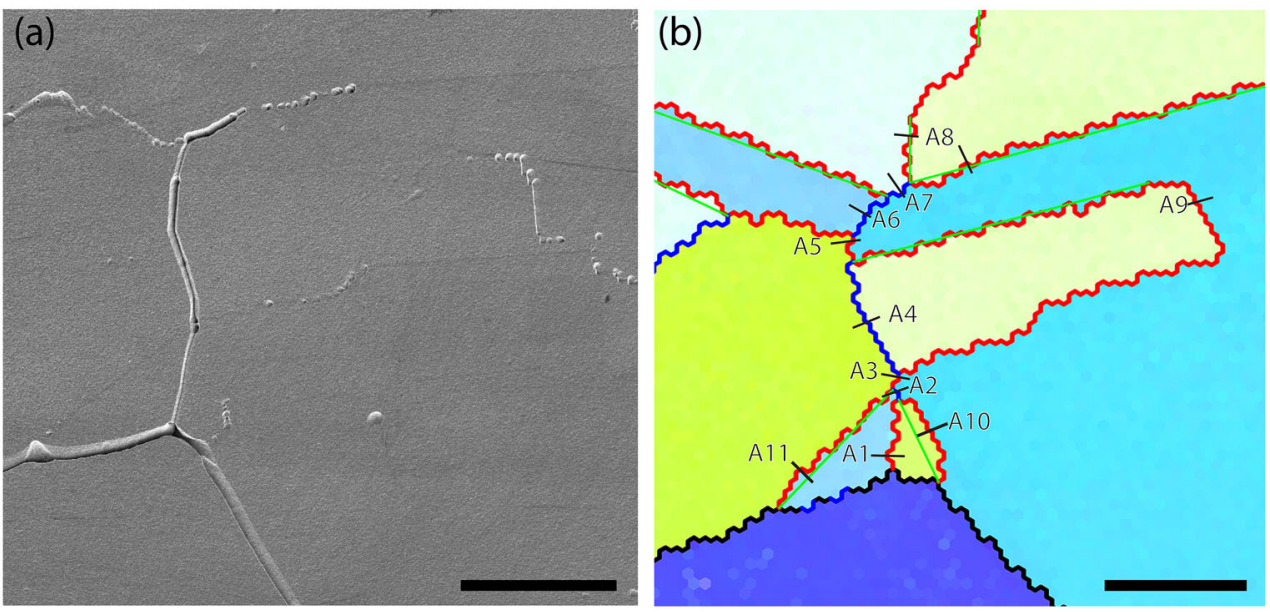

(c)

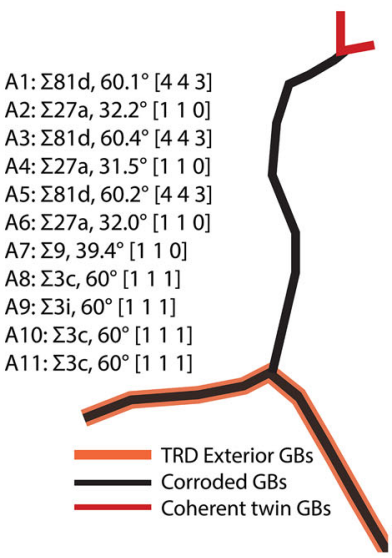

Fig. 5 a SEM image after IGC where corroded GBs show visible attack. b Corresponding inverse pole figure colored map with GB type overlaid of same location as a. c Diagram illustrating that IGC is continuous along TRD exterior GBs and has a continuous connectivity into the TRD interior at a number of higher-order twin GBs (A1-A7) until reaching a triple junction with two coherent twins (A8). Scale bar is $25 \mu \mathrm{m}$

two high-angle $\Sigma 3^{n}(n>1)$ relationships. The IGC is continuous along GBs labeled C1, C2, and C3 which are $\Sigma 243 e, \Sigma 243 a$, and $\Sigma 729 \mathrm{~b}$. In this scenario, IGC propagates throughout the TRD as it passes through a number of triple junctions that contain one coherent twin that is corrosion resistant and two higher-order twin relationship that are not corrosion resistant.

To illustrate the level of IGC on higher-order twin GBs, Table 3 provides the percentage of corroded GBs by number fraction. It provides a simplified view on the GB character level of corrosion observed in the higher-order twins ( $\Sigma 9$ and $\Sigma 27)$ and compares these values to non-twinned random low-angle GBs $\left(<15^{\circ}\right.$ misorientation) and random high-angle GBs. The level of corrosion observed between the $\Sigma 9, \Sigma 27$, and random high-angles GBs are nearly equivalent with percentages of corrode GBs between 92 and $95 \%$. Therefore, under the sensitization and corrosive environments conditions explored in this study, $\Sigma 9$ and $\Sigma 27 \mathrm{GBs}$ do not help in regard to IGC resistance inside the TRD and corrode at a rate equivalent to random GBs. Only $22 \%$ of the random lowangle GBs corrode on average. This provides further validation that the observed twin-related low-angle GBs, $\Sigma 243 \mathrm{C}$ and $\Sigma 243 \mathrm{i}$, are likely corrosion resistant because of their low misorientation angle.

Microchemistry at susceptible grain boundaries

The formation of $\mathrm{Cr}$-rich carbides and subsequent $\mathrm{Cr}$-depleted zones along GBs during sensitization is the primary factor in the reduced ability to form a continuous passive and protective oxide. To verify the GB microchemistry and precipitation, STEM-EDS was completed to illustrate the formation of $\mathrm{Cr}$-rich phases along susceptible GBs. Figure $8 a$ and $b$ indicates the microchemistry and precipitation behavior of two random high-angle GBs from the SA condition in the $1 \mathrm{~h}$ and $50 \mathrm{~h}$ sensitized condition, respectively. STEM-EDS results indicate the formation of a $\mathrm{Cr}$ and Mo-rich phase along random GBs after the $1 \mathrm{~h}$ condition. The $50 \mathrm{~h}$ sensitization condition indicates the formation of larger $\mathrm{Cr}$-rich carbides consistent with $\mathrm{M}_{23} \mathrm{C}_{6}$ formed along random GBs. Selected area diffraction, shown in Supplementary Fig. S1, confirms the structure of the $\mathrm{GB}$ precipitate as a $\mathrm{Cr}$-rich $\mathrm{M}_{23} \mathrm{C}_{6}$ phase. These large $\mathrm{Cr}$-rich precipitates and the subsequent $\mathrm{Cr}$-depletion zone formed is the primary cause for the increased corrosion rates and DOS observed in the bulk testing described above.

\section{DISCUSSION}

Correlating TRD statistics to IGC

The results indicate that IGC has a systematically different response at locations at the exterior and interior of a TRD. In the interior of TRDs, triple junctions containing twin-related low-angle GBs and coherent twins arrest IGC propagation. At the exterior of the TRDs, the random GB network was typically shown to be susceptible to IGC propagation. These observations are in general agreement with Cayron, ${ }^{28}$ which showed that TRDs are an 

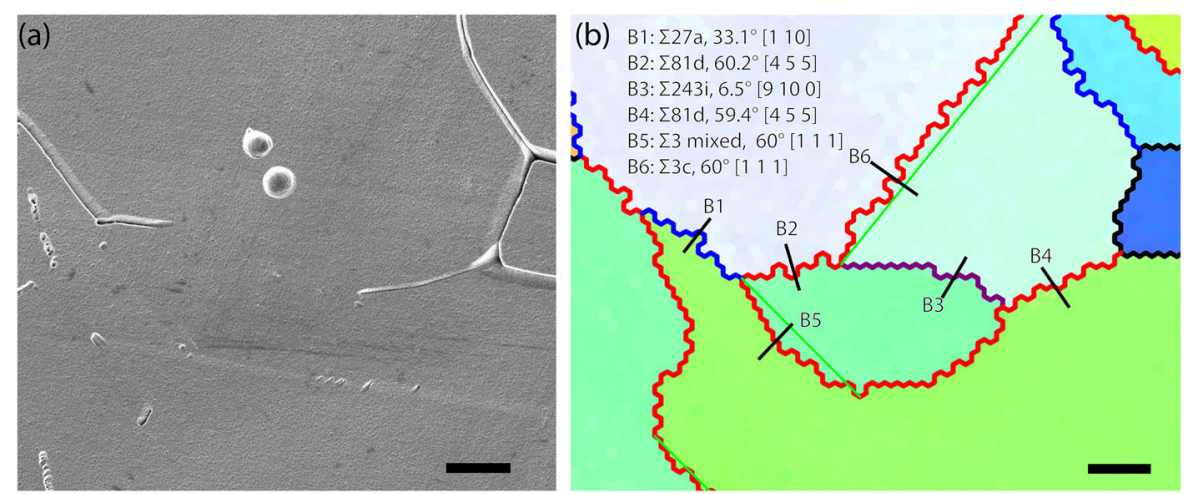

Fig. 6 a SEM image after IGC where corroded GBs show visible attack. b Corresponding inverse pole figure colored map with GB type overlaid of same location as a with GB labeled B1-B6, illustrating that IGC can be arrested at particular low-angle GBs (B3) that form during multiple twinning. Scale bar is $10 \mu \mathrm{m}$
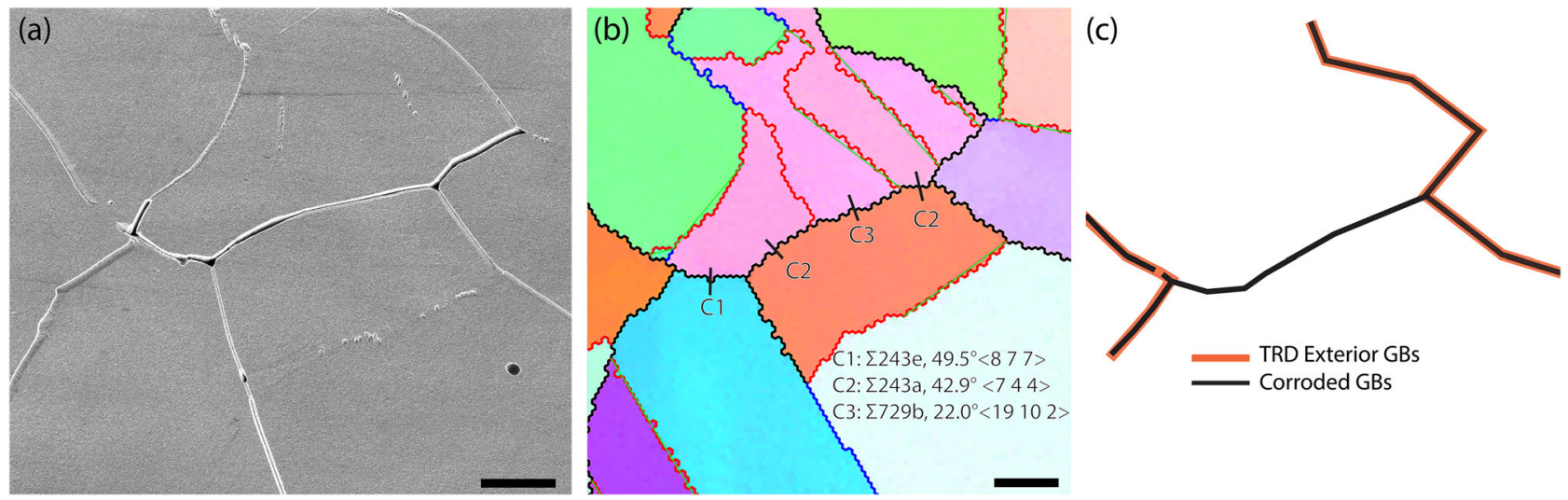

Fig. 7 a SEM image after IGC where corroded GBs show visible attack. b Corresponding inverse pole figure map with GB type overlaid of same location in a with GBs labeled C1-C3. c Diagram illustrating continuous IGC can occur through the width of a TRD where $\Sigma 243 e, \Sigma 243 a$, and $\Sigma 729 \mathrm{~b}$ are all higher-order twins but still susceptible to IGC. Scale bar is $20 \mu \mathrm{m}$

Table 3. Overview of the number percentage of GBs corroded observed at $\Sigma 9, \Sigma 27$, random high-angle GBs, and random low-angle GBs

\begin{tabular}{lll}
\hline GB type & $\begin{array}{l}\text { Corroded GBs } \\
(\%)\end{array}$ & $\begin{array}{l}\text { Total measured } \\
\text { GBs (no.) }\end{array}$ \\
\hline$\Sigma 9$ & 95 & 48 \\
$\Sigma 27$ & 93 & 45 \\
High-angle random & 92 & 176 \\
Low-angle random & 22 & 9 \\
\hline
\end{tabular}

important microstructural entity since all grains within the TRD are linked to a resistant $\Sigma 3 \mathrm{~GB}$. Therefore, intergranular phenomena such as IGC propagates between adjacent TRDs since it is typically arrested in the interior of TRDs. Since the corrosion propagation is shown to occur between adjacent TRDs, it is important to evaluate heavily twinned microstructures based on representative TRD statistics.

We propose that TRD statistics between different TMP conditions can provide insight into indicating IGC susceptible and resistant microstructures. This was highlighted by the vastly different bulk IGC behavior shown in the SA and GBE conditions. During the GBE TMP process, the size of the TRD, evaluated by TRD statistics by the average diameter $(396 \mu \mathrm{m})$ and number of unique orientations (14.1), grows significantly from the SA state as shown in Table 2. Under the assumption that IGC propagation is eventually arrested inside the TRD, a microstructure with large TRDs, such as those found in GBE conditions, will be more IGC resistant than a microstructure with small TRDs. Since corrosion propagation occurs around the TRD, the TRD size is proposed to be an effective descriptor of the overall IGC resistance. Figure $3 e$ and $f$ highlights this difference in predictive IGC behavior between GBE and non-GBE conditions due to the size difference of the TRDs. It is conspicuous that as the TRD size increases, the propagation distance for continuous percolation of IGC increases. As described in our previous work, ${ }^{23}$ a larger TRD to grain size ratio is proposed to provide a microstructure with spatially large TRDs and smaller twin-related GBs within the TRD. The GBE condition, with a three-fold increase TRD to grain size ratio, compared to the SA shows dramatically improved bulk IGC behavior. The observation that TRD size plays a critical factor in predicting IGC behavior is in agreement with grain cluster analysis of GBE and non-GBE Alloy 690 tube by Xia et al. ${ }^{37}$ where they observe increased weight loss after IGC with small grain clusters associated with non-GBE condition compared to large GBE grain clusters.

As detailed above, we propose that TRD size and subsequent statistics provide the appropriate length scale and microstructural descriptor in correlating TMP conditions and IGC phenomena. Further efforts are required to fully confirm the applicability using different TMP condition TRD statistics as an effective descriptor 

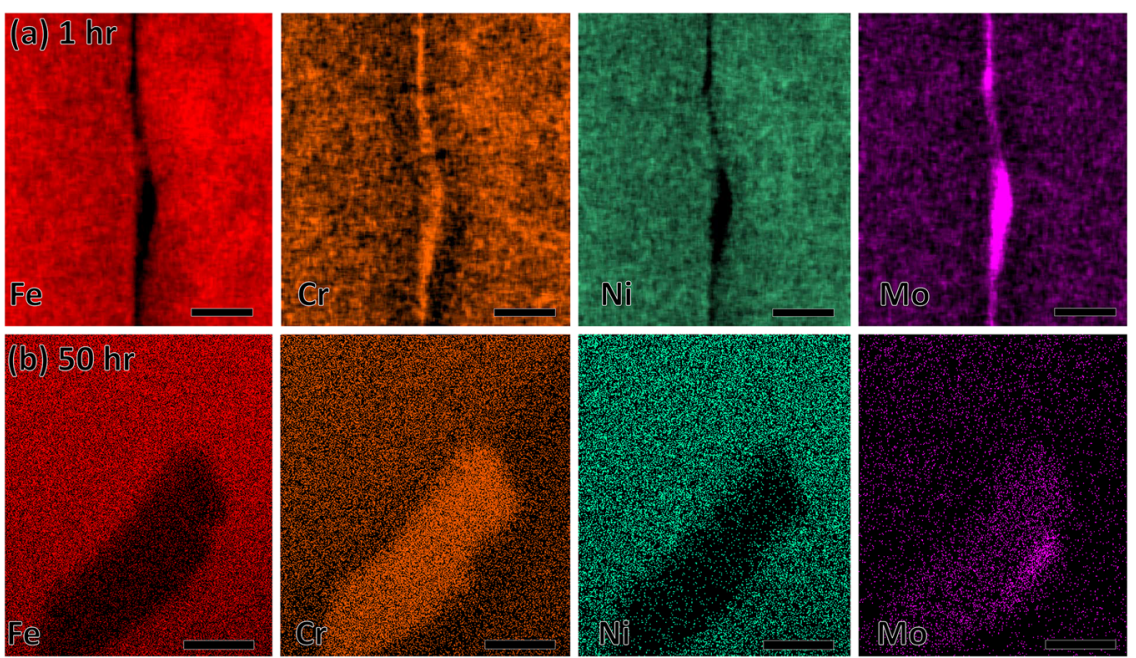

Fig. 8 Overview grain boundary microchemistry and carbide precipitation at a random high-angle GB in the SA condition for the a $1 \mathrm{~h}$ sensitized and $\mathbf{b} 50 \mathrm{~h}$ sensitized condition. Scale bar in $\mathbf{a}$ and $\mathbf{b}$ are 30 and $100 \mathrm{~nm}$, respectively

IGC phenomena. For example, a number of other microstructural parameters have been used to correlate IGC phenomena with microstructural features including a decrease in the maximum random boundary connectivity. ${ }^{38}$ Furthermore, previous studies $^{39,40}$ have indicate that there is a correlation between IGC phenomena measured by DOS and effective grain boundary energy (EGBE). EGBE is able to account both for the fraction of CSL boundaries and grain size. To fully appreciate the TRD statistics, a detailed comparison on the various microstructural descriptors that have been used to understand and predict IGC is required.

Role of GB character in IGC

Numerous computation studies ${ }^{17,41-48}$ have indicated significant anisotropy in GB properties, including GB energy, free volume, and mobility as function of the full macroscopic GB character, including both the boundary misorientation and plane. One proposed key property related to IGC is the GB energy since sensitization and subsequent IGC along particular $\mathrm{GBs}$ is dependent on the nucleation and growth of $M_{23} C_{6}$ carbide. ${ }^{1,49,50}$ Olmsted et al. ${ }^{41}$ explored the GB energy landscape of 388 distinct $\mathrm{GBs}$ in pure $\mathrm{Ni}$ and observed significant variations for between coherent and non-coherent twin GBs. Specifically, they showed that the symmetric coherent twin $\left(\sum 3<110>\{111\}\right)$ has extremely low GB energy of $0.06 \mathrm{~J} / \mathrm{m}^{2}$, while all other asymmetric and symmetric GB planes of the $\Sigma 3$ had significantly higher values, up to approximately $1 \mathrm{~J} / \mathrm{m}^{2}$. The low GB energy corroborates the lack of any corrosion attack on coherent $\Sigma 3 \mathrm{GBs}$ in our study. The large spread in GB energy in the $\Sigma 3$ GB plane space is not observed in other GBE relevant GBs such as the $\Sigma 9 \mathrm{~GB}$. Here, all observed symmetric and asymmetric $\Sigma 9 \mathrm{GBs}$ have higher $\mathrm{GB}$ energy ranging from approximately 0.8 to $1.3 \mathrm{~J} / \mathrm{m}^{2}{ }^{21}$ The high overall GB energy for all $\Sigma 9 \mathrm{GBs}$ with no sharp energy minimization cusp in pure $\mathrm{Ni}$ is consistent with our study. In our study, approximately $95 \%$ of $\Sigma 9$ GBs indicated significant IGC attack after the $1 \mathrm{~h}$ sensitization and ASTM bulk corrosion test. Furthermore, the other two corrosionresistant GBs examined besides the coherent twin GB were the low-angle twin-related $\Sigma 243 \mathrm{C}$ and $\Sigma 243 \mathrm{i}$ GBs likely have lower than average GB energy. Olmsted et al. ${ }^{41}$ showed gradual increasing GB energy from low to high misorientation angle GBs across all presented tilt and twist GBs.

It has been shown experimentally that M23C6 Cr-rich carbides and subsequent $\mathrm{Cr}$-depletion regions exist along GBs in 304/304L and $316 / 316 \mathrm{~L}$ alloys. Trillo et al. ${ }^{51,52}$ examined $\mathrm{Cr}$ carbide nucleation and growth in 304 type stainless steel and found extensive carbide growth on random high-angle GBs and incoherent twin GBs, while coherent twins provided resistance to carbide nucleation and growth. This observation is consistent with a number of similar studies in both stainless steel ${ }^{50}$ and $\mathrm{Ni}$ based Alloy $600^{53}$ which indicated that coherent twin GBs are resistant to $G B$ nucleation, while incoherent twins, $\Sigma 9, \Sigma 27$, and other higher-order twin relationships can subsequently form deleterious $\mathrm{Cr}$-rich carbides and adjacent $\mathrm{Cr}$-depleted zones. In this study, as shown in Fig. 8, the $50 \mathrm{~h}$ sensitization treatment clearly forms $\mathrm{Cr}$-rich carbides along random $\mathrm{GBs}$ and subsequent $\mathrm{Cr}$-depleted zones. The $\mathrm{Cr}$-depleted zones can subsequently be linked to the reduced bulk IGC resistance of the SA condition which contains a higher density of random GBs and smaller TRDs compared to the GBE condition.

It is important to note that the uniformity or depth of corrosion attack is different between the GBs observed in the study. In this study, there was no separation between GBs as function of corrosion depth as boundaries were only considered to either be attacked or resistant to IGC. As observed in Fig. 5, it is apparent, however, that although GBs labeled A1 through A7 all have significant IGC, the attack depth is different as function of GB type. For example, GB A1 is a $\Sigma 81 \mathrm{~d} 60.1^{\circ}<4 \quad 43>\mathrm{GB}$ which has qualitatively smaller corrosion depth attack compared to for example the $\Sigma 9$ or $\Sigma 27$ a GBs labeled A3 and A4 in Fig. 5. Recent studies $^{54,55}$ explored newer methods to examine threedimensional effects of the GB network on corrosion or stress corrosion cracking propagation by focused ion beam (FIB) serial sectioning and three-dimensional electron backscatter diffraction (EBSD) and is planned for a future follow-up study. FIB crosssectional and/or three-dimensional EBSD provide improved means to determine the full macroscopic GB description and localized GB network effects.

In conclusion, the objective of this work was to correlate and enhance the understanding of IGC phenomena observed in a GBE and SA condition with effective microstructural descriptors. It was shown that TRDs provide an effective microstructural descriptor to evaluate both individual GB-dependent corrosion propagation and overall IGC resistance. The following key finds from the study include:

- Large TRDs (measured both by an effective diameter and number of unique orientation) such as those observed in the GBE condition are shown to be resistant to IGC. The link between an increase in TRD size (GBE condition) and improved corrosion resistance is observed in both bulk scale 
corrosion testing completed: DL-EPR and mass loss testing after sensitization in 316L.

- IGC can propagate into the TRD and is only fully arrested at triple junctions containing two GBs of the coherent twins or the twin-related low-angle GBs ( $\Sigma 243 \mathrm{C}$ and $\Sigma 243 \mathrm{i}$ ).

- Propagation of IGC does not stop at any higher-order twin GBs $\left(\Sigma 3^{n}, n>1\right)$ aside from the aforementioned two low-angle $\Sigma 3^{5}$ GBs. This has important implications as IGC can propagate along triple junctions containing these higher-order twin boundaries without arresting.

- $\Sigma 3$ coherent twins are resistant to IGC while non-coherent twins behave like random high-angle GBs, which is consistent with past studies; these differences in IGC resistance are linked to the dramatically different atomic GB structure between the coherent and non-coherent twin GB.

\section{METHODS}

To examine the role of GB character and TRDs on IGC, different TMP routes were developed to obtain both standard SA and GBE microstructures. AISI 316L stainless steel was obtained from Carpenter Technology (Reading, PA) in the mill-annealed state with a nominal chemical composition given in Table 1. As-received specimens were SA for $60 \mathrm{~min}$ at $1050^{\circ} \mathrm{C}$ followed by a water quench. The GBE microstructure consisted of $5 \%$ rolling reduction of nominal $10 \mathrm{~mm}$ thick samples followed by a $1025^{\circ} \mathrm{C}$ anneal in open air for $90 \mathrm{~min}$ and water quench. ${ }^{46}$ The GBE and SA microstructures were examined by EBSD with a TexSEM Laboratories orientation imaging microscopy $\left(\mathrm{OIM}^{\circledast}\right)$ system. GB length and number population of twin and twin-variant GBs $\left(\Sigma 3^{n}\right)$ were calculated using the Brandon criterion for CSL $\mathrm{GBs}^{56}$ The classification and distribution of triple junction types were determined by the type of GBs at the triple junction following the methods described in Rohrer et al. ${ }^{57}$ and Randle et al. ${ }^{9}$ using multiple large data sets of $>50,000$ total GB segments analyzed per condition. The types of GB at a triple junction were classified as random $(R), \Sigma 3$ coherent $(\Sigma 3 \mathrm{C}), \Sigma 3$ noncoherent $(\Sigma 3 \mathrm{i}), \Sigma 9$, or $\Sigma 27$. The distribution of triple junctions accounted for the coherency of the $\Sigma 3 \mathrm{~GB}$ described in detail by Wright and Larsen. ${ }^{58}$ Briefly, we used $2 \times$ the step size $(2 \mu \mathrm{m})$ to define the maximum deviation between the reconstructed $\mathrm{GB}$ and $\mathrm{GB}$ segment and a $5^{\circ}$ trace deviation between the reconstructed GB trace and the $\{111\} \mathrm{GB}$ trace. This method provides an estimate of whether the $\Sigma 3 \mathrm{~GB}$ is a coherent or non-coherent twin segment.

TRDs were examined following the procedures described by $\operatorname{Reed}^{27,29}$ and Cayron. ${ }^{28}$ Adjacent orientations were considered part of the same TRD if they were identified as having either a $\Sigma 3$ or $\Sigma 3^{n}(n>1)$ relationship with a maximum deviation from the ideal misorientation of $2^{\circ}$. Each orientation was given a grain "ID" to track the number of distinct orientations in the TRD. Adjacent grains with higher-order twin misorientation relationship $\left(\Sigma 3^{n}\right.$, where $\left.n>3\right)$ were considered part of the same twin domain. Therefore, by this classification of a TRD, GBs located along the exterior of the TRD were considered random GBs, while all GBs inside the TRD were considered twin-related GBs.

Site-specific boiling ferric sulfate-sulfuric acid IGC mass loss test, ASTM A262 Practice $B_{1}^{59}$ was carried on both sample conditions (GBE and SA) after both $1 \mathrm{~h}$ and $50 \mathrm{~h} 675^{\circ} \mathrm{C}$ sensitization heat treatment in an open air box furnace environment, followed by air cooling. The ferric sulfate sulfuric acid test was completed in accordance with ASTM-A262-15 practices where sulfuric acid is in a solution of dissolved ferric sulfate in distilled water. Individual samples per condition with approximate size of $14 \mathrm{~mm} \times$ $14 \mathrm{~mm} \times 8 \mathrm{~mm}$ were placed in specialty made glass cradle and placed in the boiling solution. Three samples per condition were examined for bulk IGC corrosion rate for non-site-specific corrosion testing. Here, the sensitized samples of both the GBE and SA condition were examined for IGC corrosion rate $(\mathrm{mm} / \mathrm{yr})$ in accordance with ASTM A262B-15 at $120 \mathrm{~h}$ of testing using an analytical balance to measure loss in weight.

The DL-EPR test were also performed to obtain a bulk measure of the DOS between the 316L SA and GBE conditions. The procedures followed for the DL-EPR tests were similar to those followed by Srinivasan et al. ${ }^{30}$ Sensitized samples as described above were surface polished to a $1 \mu \mathrm{m}$ diamond surface finish before testing and cleaned ultrasonically. A deaerated $0.5 \mathrm{M} \mathrm{H}_{2} \mathrm{SO}_{4}+0.01 \mathrm{M}$ Potassium thiocyanate (KSCN) solution at room temperature was used as the test electrolyte for the DL-EPR tests. An electrochemical "flat cell" (manufactured by Princeton Applied Research) was used for electrochemical testing. The three-electrode configuration was used with the saturated calomel electrode (SCE) used as the reference electrode and a platinum mesh used as the counter electrode. The test electrolyte was de-aerated using Argon gas for $45 \mathrm{~min}$ prior to commencement of the electrochemical tests. The $316 \mathrm{~L}$ specimens were firstly subject to cathodic cleaning in the test electrolyte by polarization at $-1 V_{S C E}$ for $2 \mathrm{~min}$ and then allowed to stabilize in the same electrolyte for $15 \mathrm{~min}$. The specimens were then polarized from $-0.45 V_{\mathrm{SCE}}$ to $0.3 V_{\mathrm{SCE}}$ in the anodic direction and then reversed at $0.3 V_{S C E}$ back toward $-0.45 V_{S C E}$ in the cathodic direction. The scan rate used was $6 \mathrm{~V} / \mathrm{h}$. A peak current density was attained both during the forward scan (activation scan) and also the reverse scan (reactivation scan). The DOS can be estimated by Eq. 1 , where $I_{\mathrm{r}}$ is the maximum current density on reactivation scan (reverse scan) and $l_{\mathrm{a}}$ is the maximum current density on the activation scan (forward scan).

$\operatorname{DOS}=\left(\frac{l_{\mathrm{r}}}{l_{\mathrm{a}}}\right) \times 100$.

To carry out the site-specific IGC testing, both SA and GBE specimens were vibropolished perpendicular to the rolling direction and labeled with fiducial marks on the surface; SEM-EBSD analysis was completed in the area of interest before IGC. The remaining sample faces were prepared to a 600 grit (P1200) surface finish. Following $24 \mathrm{~h}$ of IGC testing, SEM was used to examine the regions of interest from the pre-IGC EBSD area surrounding the fiducial marks. The pre-corrosion EBSD region was used to allow a comparison of the character of GBs that were corroded.

Samples for scanning transmission electron microscopy (STEM) were prepared from the SA as-sensitized 1 and $50 \mathrm{~h}$, condition. GB site-specific samples were prepared by standard FIB cross-sectional lift-out methods in a FEI Stratus DB235 FIB/SEM. Site-specific GBs were determined in the FIB/ SEM by determining the GB character by EBSD. STEM-energy dispersive $X-$ ray spectroscopy (EDS) maps and line scans were completed using either a FEl Talos F200X field-emission analytical TEM/STEM at $200 \mathrm{kV}$ or a JEOL 2100 field-emission analytical microscope. For EDS, only Fe, Cr, Ni, Si, Mo were considered during analysis as $C$ cannot be accurately quantified by EDS analysis. ${ }^{60}$

Data availability

The data that supports the results of this study can be made available from the corresponding author upon reasonable request.

\section{ACKNOWLEDGEMENTS}

C.M.B. and M.L.T. acknowledge technical assistance in corrosion testing from Thomas Worley, Carpenter Technology (Reading, PA). C.M.B., J.L.H., W.H., E.A., and M.L.T. acknowledge funding from NSF Early Career Award Program (Grant \#115087). Yao Qiu and Shravan Kairy are gratefully acknowledged for their technical assistance with the DL-EPR tests.

\section{AUTHOR CONTRIBUTIONS}

C.M.B. and M.L.T. planned the work and wrote initial manuscript. C.M.B. performed and oversaw the bulk mass loss corrosion experiments, alloy processing, SEM and EBSD collection, and all data analysis. E.A. assisted in the data analysis. W.H. and J.L.H. assisted in the GB TEM image collection and analysis. S.T. performed and oversaw the DL-EPR corrosion testing. All co-authors contributed and reviewed the finalized manuscript.

\section{ADDITIONAL INFORMATION}

Supplementary information accompanies the paper on the npj Materials Degradation website (https://doi.org/10.1038/s41529-018-0032-7).

Competing interests: The authors declare no competing interests.

Publisher's note: Springer Nature remains neutral with regard to jurisdictional claims in published maps and institutional affiliations.

\section{REFERENCES}

1. Hall, E. L. \& Briant, C. L. Chromium depletion in the vicinity of carbides in sensitized austenitic stainless steels. Metall. Trans. A 15, 793-811 (1984).

2. Pande, C. S., Suenaga, M., Vyas, B., Isaacs, H. S. \& Harling, D. F. Direct evidence of chromium depletion near the grain boundaries in sensitized stainless steels. Scr. Metall. 11, 681-684 (1977). 
3. Chen, A. Y. et al. Improving the intergranular corrosion resistance of austenitic stainless steel by high density twinned structure. Scr. Mater. 130, 264-268 (2017).

4. Randle, V. Twinning-related grain boundary engineering. Acta Mater. 52, 4067-4081 (2004).

5. Hu, C. et al. Improving the intergranular corrosion resistance of 304 stainless steel by grain boundary network control. Corros. Sci. 53, 1880-1886 (2011).

6. Michiuchi, M., Kokawa, H., Wang, Z. J., Sato, Y. S. \& Sakai, K. Twin-induced grain boundary engineering for 316 austenitic stainless steel. Acta Mater. 54, 5179-5184 (2006).

7. Schuh, C. A., Kumar, M. \& King, W. E. Analysis of grain boundary networks and their evolution during grain boundary engineering. Acta Mater. 51, 687-700 (2003).

8. Kumar, M., Schwartz, A. J. \& King, W. E. Microstructural evolution during grain boundary engineering of low to medium stacking fault energy fcc materials. Acta Mater. 50, 2599-2612 (2002).

9. Randle, V., Rohrer, G. S., Miller, H. M., Coleman, M. \& Owen, G. T. Five-parameter grain boundary distribution of commercially grain boundary engineered nickel and copper. Acta Mater. 56, 2363-2373 (2008).

10. Shi, F. et al. Improving intergranular corrosion resistance in a nickel-free and manganese-bearing high-nitrogen austenitic stainless steel through grain boundary character distribution optimization. Corros. Sci. 107, 49-59 (2016).

11. Gertsman, V. Y. \& Bruemmer, S. M. Study of grain boundary character along intergranular stress corrosion crack paths in austenitic alloys. Acta Mater. 49, 1589-1598 (2001).

12. Telang, A. et al. Iterative thermomechanical processing of alloy 600 for improved resistance to corrosion and stress corrosion cracking. Acta Mater. 113, 180-193 (2016).

13. Tsurekawa, S., Nakamichi, S. \& Watanabe, T. Correlation of grain boundary connectivity with grain boundary character distribution in austenitic stainless steel. Acta Mater. 54, 3617-3626 (2006).

14. Shimada, M., Kokawa, H., Wang, Z., Sato, Y. \& Karibe, I. Optimization of grain boundary character distribution for intergranular corrosion resistant 304 stainless steel by twin-induced grain boundary engineering. Acta Mater. 50, 2331-2341 (2002).

15. Bechtle, S., Kumar, M., Somerday, B. P., Launey, M. E. \& Ritchie, R. O. Grain boundary engineering markedly reduces susceptibility to intergranular hydrogen embrittlement in metallic materials. Acta Mater. 57, 4148-4157 (2009).

16. Schlegel, S. M., Hopkins, S. \& Frary, M. Effect of grain boundary engineering on microstructural stability during annealing. Scr. Mater. 61, 88-91 (2009).

17. Homer, E. R., Patala, S. \& Priedeman, J. L. Grain boundary plane orientation fundamental zones and structure-property relationships. Sci. Rep. 5, 15476 (2015).

18. Lind, J., Li, S. F. \& Kumar, M. Twin related domains in 3D microstructures of conventionally processed and grain boundary engineered materials. Acta Mater. 114, 43-53 (2016).

19. Bober, D. B., Lind, J., Mulay, R. P., Rupert, T. J. \& Kumar, M. The formation and characterization of large twin related domains. Acta Mater. 129, 500-509 (2017).

20. Mason, J. K. et al. Statistics of twin-related domains and the grain boundary network. Acta Mater. 61, 6524-6532 (2013).

21. Irukuvarghula, S. et al. Acta materialia evolution of grain boundary network topology in $316 \mathrm{~L}$ austenitic stainless steel during powder hot isostatic pressing. Acta Mater. 133, 269-281 (2017).

22. Leff, A., Nye, A., Barr, C. M. \& Taheri, M. L. Direct observation of recrystallization mechanisms during annealing of $\mathrm{Cu}$ in low and high strain conditions. Scr. Mater. 146, 308-311 (2018).

23. Barr, C. M., Leff, A. C., Demott, R. W., Doherty, R. D. \& Taheri, M. L. Unraveling the origin of twin related domains and grain boundary evolution during grain boundary engineering. Acta Mater. 144, 281-291 (2018).

24. Gertsman, V. Y. \& Henager, C. H. Grain boundary junctions in microstructure generated by multiple twinning. Interface Sci. 11, 403-415 (2003).

25. Fang, X., Zhang, K., Guo, H., Wang, W. \& Zhou, B. Twin-induced grain boundary engineering in 304 stainless steel. Mater. Sci. Eng. A 487, 7-13 (2008).

26. Xia, S. \& Zhou, B. \& Chen, W. Grain cluster microstructure and grain boundary character distribution in alloy 690. Metall. Mater. Trans. A 40, 3016-3030 (2009).

27. Reed, B. W. \& Kumar, M. Mathematical methods for analyzing highly-twinned grain boundary networks. Scr. Mater. 54, 1029-1033 (2006).

28. Cayron, C. Quantification of multiple twinning in face centred cubic materials. Acta Mater. 59, 252-262 (2011)

29. Reed, B. W., Kumar, M., Minich, R. W. \& Rudd, R. E. Fracture roughness scaling and its correlation with grain boundary network structure. Acta Mater. 56, 3278-3289 (2008).

30. Srinivasan, N. et al. Near boundary gradient zone and sensitization control in austenitic stainless steel. Corros. Sci. 100, 544-555 (2015).

31. Rahimi, S., Engelberg, D. L. \& Marrow, T. J. A new approach for DL-EPR testing of thermo-mechanically processed austenitic stainless steel. Corros. Sci. 53, 4213-4222 (2011).
32. Aydogdu, G. H. \& Aydinol, M. K. Determination of susceptibility to intergranular corrosion and electrochemical reactivation behaviour of AISI 316L type stainless steel. Corros. Sciecne 48, 3565-3583 (2006).

33. Momeni, M., Hadi, M. \& Davoodi, A. Tuning DOS measuring parameters based on double-loop EPR in $\mathrm{H} 2$ SO 4 containing KSCN by Taguchi method. Corros. Sci. 52, 2653-2660 (2010).

34. Randle, V. \& Jones, R. Grain boundary plane distributions and single-step versus multiple-step grain boundary engineering. Mater. Sci. Eng. A 524, 134-142 (2009).

35. Jones, R. \& Randle, V. Sensitisation behaviour of grain boundary engineered austenitic stainless steel. Mater. Sci. Eng. A 527, 4275-4280 (2010).

36. Gottstein, G. Annealing texture development by multiple twinning in F.C.C. crystals. Acta Metall. 32, 1117-1138 (1984).

37. Xia, S., Li, H., Liu, T. G. \& Zhou, B. X. Appling grain boundary engineering to Alloy 690 tube for enhancing intergranular corrosion resistance. J. Nucl. Mater. 416 303-310 (2011).

38. Kobayashi, S., Kobayashi, R. \& Watanabe, T. Control of grain boundary connectivity based on fractal analysis for improvement of intergranular corrosion resistance in SUS316L austenitic stainless steel. Acta Mater. 102, 397-405 (2016).

39. Wasnik, D. N., Kain, V., Samajdar, I., Verlinden, B. \& De, P. K. Resistance to sensitization and intergranular corrosion through extreme randomization of grain boundaries. Acta Mater. 50, 4587-4601 (2002).

40. Parvathavarthini, N. et al. Sensitization control in AISI $316 \mathrm{~L}(\mathrm{~N})$ austenitic stainless steel: defining the role of the nature of grain boundary. Corros. Sci. 51, 2144-2150 (2009).

41. Olmsted, D. L., Foiles, S. M. \& Holm, E. A. Survey of computed grain boundary properties in face-centered cubic metals: I. Grain boundary energy. Acta Mater. 57, 3694-3703 (2009).

42. Tschopp, M. A. \& Mcdowell, D. L. Asymmetric tilt grain boundary structure and energy in copper and aluminium. Philos. Mag. 87, 3871-3892 (2007).

43. Tschopp, M. A. et al. Probing grain boundary sink strength at the nanoscale: energetics and length scales of vacancy and interstitial absorption by grain boundaries in a-Fe. Phys. Rev. B 85, 1-21 (2012).

44. Brown, J. \& Ghoniem, N. Structure and motion of junctions between coherent and incoherent twin boundaries in copper. Acta Mater. 57, 4454-4462 (2009).

45. Wolf, D. Structure-energy correlation for grain boundaries in F.C.C. metals-III. Symmetrical tilt boundaries. Acta Met. Mater. 38, 781-790 (1990).

46. Barr, C. M. et al. Anisotropic radiation induced segregation in $316 \mathrm{~L}$ austenitic stainless steel with grain boundary character. Acta Mater. 67, 145-155 (2014).

47. Ratanaphan, S. et al. Grain boundary energies in body-centered cubic metals. Acta Mater. 88, 346-354 (2015).

48. Bulatov, V. V., Reed, B. W. \& Kumar, M. Grain boundary energy function for fcc metals. Acta Mater. 65, 161-175 (2014).

49. Li, H., Xia, S., Liu, W., Liu, T. \& Zhou, B. Atomic scale study of grain boundary segregation before carbide nucleation in Ni-Cr-Fe alloys. J. Nucl. Mater. 439, 57-64 (2013)

50. Laws, M. S. \& Goodhew, P. Grain boundary structure and chromium segregation in a 316 stainless steel. Acta Metall. Mater. 39, 1525-1533 (1991).

51. Trillo, E. A. \& Murr, L. E. A TEM investigation of M23C6 carbide precipitation behaviour on varying grain boundary misorientations in 304 stainless steels. J. Mater. Res. 33, 1263-1271 (1998).

52. Trillo, E. A. \& Murr, L. E. Effects of carbon content, deformation, and interfacial energetics on carbide precipitation and corrosion sensitization in 304 stainless steel. Acta Mater. 47, 235-245 (1999).

53. $\mathrm{Li}, \mathrm{H}$. Twinning structure of $\mathrm{M} 23 \mathrm{C} 6$ carbide precipitated at twin-related grain boundaries in Alloy 600. Philos. Mag. 96, 551-559 (2016).

54. Stratulat, A., Duff, J. A. \& Marrow, T. J. Grain boundary structure and intergranular stress corrosion crack initiation in high temperature water of a thermally sensitised austenitic stainless steel, observed in situ. Corros. Sci. 85, 428-435 (2014).

55. Liu, T. et al. The topology of three-dimensional grain boundary network and its influence on stress corrosion crack propagation characteristics in austenitic stainless steel in a simulated BWR environment. Corros. Sci. 129, 161-168 (2017).

56. Brandon, D. E. The structure of high-angle grain boundaries. Acta Metall. 14, 1479-1484 (1966).

57. Rohrer, G. S., Randle, V., Kim, C.-S. \& Hu, Y. Changes in the five-parameter grain boundary character distribution in a-brass brought about by iterative thermomechanical processing. Acta Mater. 54, 4489-4502 (2006).

58. Wright, S. I. \& Larsen, R. J. Extracting twins from orientation imaging microscopy scan data. J. Microsc. 205, 245-252 (2002).

59. ASTM. ASTM A262-13 standard practices for detecting susceptibility to intergranular attack in austenitic stainless steel. ASTM International (ASTM International, 2013).

60. Williams, D. B. \& Carter, C. B. Transmission Electron Microscopy: A Textbook for Materials Science. (Springer). 
This article is licensed under a Creative Commons Attribution 4.0 International License, which permits use, sharing, adaptation, distribution and reproduction in any medium or format, as long as you give appropriate credit to the original author(s) and the source, provide a link to the Creative Commons license, and indicate if changes were made. The images or other third party material in this article are included in the article's Creative Commons license, unless indicated otherwise in a credit line to the material. If material is not included in the article's Creative Commons license and your intended use is not permitted by statutory regulation or exceeds the permitted use, you will need to obtain permission directly from the copyright holder. To view a copy of this license, visit http://creativecommons. org/licenses/by/4.0/.

(c) The Author(s) 2018 